\title{
Second- and third-harmonic generation with vector Gaussian beams
}

\author{
Silvia Carrasco \\ Department of Chemistry and Chemical Biology, Harvard University, Cambridge, Massachusetts 02138 \\ Bahaa E. A. Saleh and Malvin C. Teich \\ Department of Electrical and Computer Engineering, Boston University, Boston, Massachusetts 02215 \\ John T. Fourkas \\ Department of Chemistry and Biochemistry, University of Maryland, College Park, Maryland 20742
}

\begin{abstract}
Received February 21, 2006; revised June 12, 2006; accepted June 17, 2006; posted June 30, 2006 (Doc. ID 68127)
We consider second-harmonic generation (SHG) and third-harmonic generation (THG) in a nonlinear optical crystal illuminated by a vector Gaussian beam, i.e., a Gaussian beam in which the axial component of the excitation field is considered. This component exhibits twice the Gouy phase shift of the transverse component and vanishes at points on the beam axis. Harmonic generation stemming from this component exhibits a unique dependence on geometrical factors associated with the location and focusing of the beam relative to the location of the crystal. Using the first Born approximation (undepleted fundamental beam), we derive analytical formulas for the quantities that characterize these geometrical factors for a nonlinear optical crystal described by an arbitrary nonlinear susceptibility tensor, for both SHG and THG and for all polarization components. We also determine the efficiencies of these processes as functions of the geometry of the experimental arrangement for phase-matched crystals as well as for crystals of infinite length. (C) 2006 Optical Society of America
\end{abstract}

OCIS codes: $190.2620,190.4410$.

\section{INTRODUCTION}

Calculations involving harmonic generation in secondand third-order nonlinear optical media generally assume that the wave at the fundamental excitation frequency is a Gaussian beam in the $\operatorname{TEM}(0,0)$ mode. $^{1-5}$ Under these conditions, analytical expressions for the efficiencies of second-harmonic generation (SHG) and third-harmonic generation (THG) are well known; the optimal placement of the beam center within the nonlinear crystal has been determined, as has the optimal ratio of the depth of focus to the crystal length (see, for example, Ref. 6). Since harmonic generation is a coherent process, the on-axis phase of the Gaussian beam (the Gouy phase ${ }^{7,8}$ ) plays an important role in the efficiency of harmonic generation. Indeed, this phase can lead to a significant reduction in the efficiency of THG as a result of destructive interference between third-harmonic waves generated at opposite sides of the beam waist in the nonlinear crystal. Studies in imaging and microscopy systems that make use of $\mathrm{SHG}^{9-11}$ and $\mathrm{THG}^{12-17}$ have created renewed interest in the optimization of harmonic generation, and numerical techniques have been used to model this process. ${ }^{18,19}$

In previous analytical studies of harmonic generation, the fundamental Gaussian beam has always been assumed to be scalar in nature, implying that the polarization is uniform across the beam. This assumption is clearly an approximation since it is inconsistent with the curvature of the beam wavefront. Since the local polarization must be orthogonal to the local direction of the wave vector, at off-axis positions the polarization must have an axial component, i.e., a component parallel to the beam axis. Since the wavefront curvature is maximal at a Rayleigh distance from the beam center, the axial component of the field is largest in this vicinity. Because this region of the beam contributes strongly to harmonic generation, the role that the axial component of the field plays in harmonic generation is worthy of consideration. A recent numerical study of SHG microscopy ${ }^{19}$ has shown that the axial field component plays a significant role in SHG from materials with certain susceptibility tensors, and it was noted that this component can be responsible for the generation of radially polarized light with linearly polarized excitation.

In this paper we consider SHG and THG when the excitation is a vector Gaussian beam, i.e., a Gaussian beam for which the axial component of the field is taken into account. $^{20}$ The axial component exhibits twice the Gouy shift of the orthogonal component and vanishes at points on the beam axis. Harmonic generation stemming from the axial component of the excitation field therefore exhibits a unique dependence on geometrical factors that characterize the location and focusing of the beam relative to the nonlinear crystal. We derive analytical formulas for factors that describe the dependence of the efficiency of SHG and THG on the geometry for all polarization components, for crystals characterized by an arbitrary nonlinear susceptibility tensor. ${ }^{21}$ As a caveat, we point out that our model for the vector Gaussian beam 
is strictly valid only in the paraxial approximation. Our approach is therefore not applicable for highly focused beams, but it nevertheless provides analytical results that are qualitatively correct in that case and therefore serve to elucidate the roles played by various geometrical factors in harmonic generation efficiencies for different polarization components.

\section{VECTOR GAUSSIAN BEAM}

The scalar Gaussian beam has a complex amplitude ${ }^{21}$ :

$$
G(\rho, z, k)=\frac{j z_{0}}{z+j z_{0}} \exp \left(-j k \frac{\rho^{2}}{2\left(z+j z_{0}\right)}\right) \exp (-j k z),
$$

where $\rho=\sqrt{x^{2}+y^{2}}$ ( $x$ and $y$ are coordinates transverse to the beam axis), $k=n \omega / c$ is the wavenumber at angular frequency $\omega$, and $n$ is the refractive index of the medium; $z_{0}=\pi n W_{0}^{2} / \lambda$ is the Rayleigh range, $W_{0}$ is the beam width, and $\lambda=2 \pi c / \omega$ is the wavelength in free space. In this expression, the amplitude at the beam center $(z=\rho=0)$ is taken to be unity. The vector field associated with this Gaussian beam has a complex amplitude

$$
\mathbf{E}^{(\omega)}(\rho, z)=G(\rho, z, k)\left[-\alpha \hat{\mathbf{x}}-\beta \hat{\mathbf{y}}+\frac{\alpha x+\beta y}{z+j z_{0}} \hat{\mathbf{z}}\right],
$$

where $\alpha$ and $\beta$ are complex parameters that determine the polarization state and the careted quantities are unit vectors. The transverse and axial components of this field are thus related to the scalar Gaussian function $G(\rho, z, k)$ by

$$
\begin{gathered}
E_{x}^{\omega}=-\alpha G(\rho, z, k), \\
E_{y}^{\omega}=-\beta G(\rho, z, k), \\
E_{z}^{\omega}=G(\rho, z, k) \frac{\alpha x+\beta y}{z+j z_{0}} .
\end{gathered}
$$

It is clear that the transverse components are scalar Gaussian beams. However, the axial component, which was ignored in prior studies of harmonic generation with Gaussian beams, is a weighted superposition of HermiteGaussian modes of order $(1,0)$ and $(0,1)$. If $\alpha$ and $\beta$ have equal phases, then $E_{x}^{\omega}$ and $E_{y}^{\omega}$ constitute a linearly polarized wave and $E_{z}^{\omega}$ is a Hermite-Gaussian mode of order $(1,0)$ with its nodal axis along the direction of the in-plane polarization vector. In general, $E_{z}^{\omega}$ has a transverse distribution with odd symmetry, i.e., $E_{z}^{\omega}(-x,-y)=-E_{z}^{\omega}(x, y)$, and vanishes at points on the beam axis $(x=y=0)$.

The ratio of the peak magnitudes of the axial and the transverse components is proportional to the beam divergence angle $\theta_{0}=W_{0} / z_{0}$. For example, for a linearly polarized beam with $\alpha=1$ and $\beta=0$, the transverse component has a peak magnitude equal to $G_{0}$ at $x=y=0$, where $G_{0}$ $=1 / \sqrt{1+\left(z / z_{0}\right)^{2}}$. The axial component has a peak magnitude equal to $0.43 \theta_{0} G_{0}$ at $x=W(z) / \sqrt{2}$ and $y=0$, where $W(z)=W_{0} \sqrt{1+\left(z / z_{0}\right)^{2}}$ is the beam width at a distance $z$ from the beam center. This magnitude is a factor of $0.43 \theta_{0}$ smaller than the peak magnitude of the transverse component, which was assumed to be unity.
The axial component has a Gouy phase of $2 \tan ^{-1}\left(z / z_{0}\right)$, which is twice that of the ordinary Gaussian beam. ${ }^{21}$ It is therefore expected that the dependence of the efficiency of SHG and THG created by the axial component will differ from those associated with the orthogonal Gaussian components.

\section{SECOND- AND THIRD-HARMONIC GENERATION}

We now consider the propagation of the vector Gaussian beam through a nonlinear crystal of length $L$ with arbitrary second-and third-order nonlinear susceptibility tensors. Two geometrical configurations are considered: In the first, the crystal is placed at the beam center, so that it extends between the planes $z=-L / 2$ and $z=+L / 2$, as illustrated in Fig. 1(a). In the second configuration, the edge of the crystal is placed at the beam center, so that the crystal extends from $z=0$ to $z=L$, as illustrated in Fig. 1(b). The extent of the crystal in the lateral plane is assumed to be much greater than the beam width so that the crystal may be assumed to be infinite in the transverse direction. In both cases, we shall see that the ratio $\xi=L / 2 z_{0}$ is a key geometrical parameter that governs the efficiencies of harmonic generation.

We now use a first Born approximation (undepleted fundamental beam) to derive expressions for the various polarization components of the generated second- and third-harmonic beams. ${ }^{22}$

For a crystal with second- and third-order nonlinear tensors defined by $d_{i j k}^{(2)}$ and $\chi_{i j k l}^{(3)}$, respectively, the fundamental Gaussian beam generates polarization densities given by

$$
\begin{aligned}
& P_{i}^{(2)}=\sum_{j k} d_{i j k}^{(2)} E_{j}^{\omega} E_{k}^{\omega}, \quad i, j, k=x, y, z, \\
& P_{i}^{(3)}=\sum_{j k l} \chi_{i j k l}^{(3)} E_{j}^{\omega} E_{k}^{\omega} E_{l}^{\omega}, \quad i, j, k, l=x, y, z,
\end{aligned}
$$

respectively, where both $d_{i j k}^{(2)}$ and $\chi_{i j k l}^{(3)}$ tensors are invariant to any permutation of their indices. These polarization densities, in turn, create sources of radiation at the second and third harmonic:

(a)

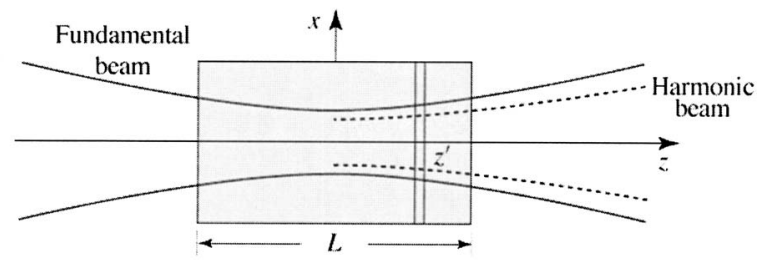

(b)

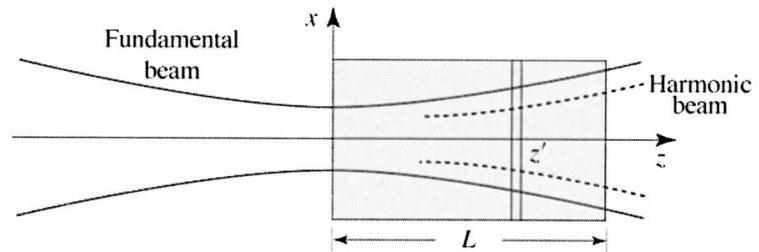

Fig. 1. Geometries for harmonic generation: (a) centered crystal, (b) offset crystal. 


$$
\begin{aligned}
& S_{i}^{(2)}=-\mu_{o} \omega^{2} \sum_{j k} d_{i j k}^{(2)} E_{j}^{\omega} E_{k}^{\omega}, \\
& S_{i}^{(3)}=-\mu_{o} \omega^{2} \sum_{j k l} \chi_{i j k l}^{(3)} E_{j}^{\omega} E_{k}^{\omega} E_{l}^{\omega},
\end{aligned}
$$

respectively.

For the vector fundamental Gaussian beam, Eqs. (5a) and $(5 \mathrm{~b})$ reduce to

$$
\begin{aligned}
S_{i}^{(2)}= & -\mu_{o} \omega^{2}\left[d_{i x x}^{(2)} S_{x x}+d_{i y y}^{(2)} S_{y y}+d_{i x y}^{(2)} S_{x y}+d_{i z z}^{(2)} S_{z z}+d_{i x z}^{(2)} S_{x z}\right. \\
& \left.+d_{i y z}^{(2)} S_{y z}\right], \\
S_{i}^{(3)}= & -\mu_{o} \omega^{2}\left[\chi_{i x x x}^{(3)} S_{x x x}+\chi_{i y y y}^{(3)} S_{y y y}+\chi_{i x y y}^{(3)} S_{x y y}+\chi_{i y x x}^{(3)} S_{y x x}\right. \\
& +\chi_{i z z z}^{(3)} S_{z z z}+\chi_{i x z z}^{(3)} S_{x z z}+\chi_{i y z z}^{(3)} S_{y z z}+\chi_{i x x z}^{(3)} S_{x x z}+\chi_{i y y z}^{(3)} S_{y y z} \\
& \left.+\chi_{i x y z}^{(3)} S_{x y z}\right],
\end{aligned}
$$

where

$$
\begin{aligned}
S_{x x}= & E_{x}^{\omega} E_{x}^{\omega}, \quad S_{y y}=E_{y}^{\omega} E_{y}^{\omega}, \quad S_{z z}=E_{z}^{\omega} E_{z}^{\omega}, \quad S_{x y}=2 E_{x}^{\omega} E_{y}^{\omega}, \\
& S_{x z}=2 E_{x}^{\omega} E_{z}^{\omega}, \quad S_{y z}=2 E_{y}^{\omega} E_{z}^{\omega}, \\
S_{x x x}= & E_{x}^{\omega} E_{x}^{\omega} E_{x}^{\omega}, \quad S_{y y y}=E_{y}^{\omega} E_{y}^{\omega} E_{y}^{\omega}, \quad S_{z z z}=E_{z}^{\omega} E_{z}^{\omega} E_{z}^{\omega} \\
S_{x y y}= & 3 E_{x}^{\omega} E_{y}^{\omega} E_{y}^{\omega}, \quad S_{y x x}=3 E_{x}^{\omega} E_{x}^{\omega} E_{y}^{\omega}, \quad S_{x z z}=3 E_{x}^{\omega} E_{z}^{\omega} E_{z}^{\omega} \\
S_{y z z}= & 3 E_{y}^{\omega} E_{z}^{\omega} E_{z}^{\omega}, \quad S_{z x x}=3 E_{x}^{\omega} E_{x}^{\omega} E_{z}^{\omega}, \quad S_{z y y}=3 E_{y}^{\omega} E_{y}^{\omega} E_{z}^{\omega} \\
S_{x y z}= & 3 E_{x}^{\omega} E_{y}^{\omega} E_{z}^{\omega} .
\end{aligned}
$$

Each of the terms of Eq. (6a) is a source of radiation at the second harmonic. Likewise, each of the terms of Eq. (6b) is a source of radiation at the third harmonic.

An example is provided by an isotropic nonlinear medium, where there are only 21 nonzero elements, of which only three are independent:

$$
\begin{aligned}
& \chi_{y y z z}^{(3)}=\chi_{z z y y}^{(3)}=\chi_{z z x x}^{(3)}=\chi_{x x z z}^{(3)}=\chi_{x x y y}^{(3)}=\chi_{y y x x}^{(3)}, \\
& \chi_{y z y z}^{(3)}=\chi_{z y z y}^{(3)}=\chi_{z x z x}^{(3)}=\chi_{x z x z}^{(3)}=\chi_{x y x y}^{(3)}=\chi_{y x y x}^{(3)}, \\
& \chi_{y z z y}^{(3)}=\chi_{z y y z}^{(3)}=\chi_{z x x z}^{(3)}=\chi_{x x z z}^{(3)}=\chi_{x y y x}^{(3)}=\chi_{y x x y}^{(3)}, \\
& \chi_{x x x x}^{(3)}=\chi_{y y y y}^{(3)}=\chi_{z z z z}^{(3)}=\chi_{x x y y}^{(3)}+\chi_{x y x y}^{(3)}+\chi_{x y y x}^{(3)}
\end{aligned}
$$

In this case the third-harmonic sources given by Eq. (6b) reduce to ${ }^{19}$

$$
\begin{aligned}
& S_{x}^{(3)}=-\mu_{o} \omega^{2} \chi_{x x x x}^{(3)}\left[S_{x x x}+\frac{1}{3} S_{x y y}+\frac{1}{3} S_{x z z}\right], \\
& S_{y}^{(3)}=-\mu_{o} \omega^{2} \chi_{y y y y}^{(3)}\left[S_{y y y}+\frac{1}{3} S_{y x x}+\frac{1}{3} S_{y z z}\right], \\
& S_{z}^{(3)}=-\mu_{o} \omega^{2} \chi_{z z z z}^{(3)}\left[S_{z z z}+\frac{1}{3} S_{z x x}+\frac{1}{3} S_{z y y}\right] .
\end{aligned}
$$

We now determine the spatial distribution of the field radiated by each of these terms, assuming that the relevant tensor coefficient is unity and ignoring the proportionality factor $\left(-\mu_{o} \omega^{2}\right)$. The calculated fields must therefore be multiplied by this factor and by the appropriate tensor element for a given crystal. A principal goal is to illustrate explicitly the dependence of each of these terms on geometrical factors such as the parameter $\xi=L / 2 z_{0}$ and the location of the beam waist (see the configurations in Fig. 1), rather than determining actual numerical values of the radiated field.

We proceed by determining each of the radiated harmonics for each of the sources in Eqs. (6a) and (6b) when the fundamental field is a vector Gaussian beam. In accordance with Fresnel diffraction theory, a source $S\left(\rho^{\prime}, z^{\prime}\right)$ at frequency $m \omega$, with $m=2$ and 3 for SHG and THG, respectively, located in the plane $z^{\prime}$, radiates a field

$$
\begin{aligned}
E^{(m \omega)}\left(x, y, z, z^{\prime}\right) \propto & h_{0}\left(z-z^{\prime}\right) \iint S\left(x^{\prime}, y^{\prime}, z^{\prime}\right) \\
& \times \exp \left(-j k_{m} \frac{\left(x-x^{\prime}\right)^{2}+\left(y-y^{\prime}\right)^{2}}{2\left(z-z^{\prime}\right)}\right) \mathrm{d} x^{\prime} \mathrm{dy}^{\prime},
\end{aligned}
$$

where $h_{0}(z)=\left(k_{m} / z\right) \exp \left(-j k_{m} z\right)$, and $k_{m}$ is the wavenumber at the $m$ th harmonic. The total radiated field in the $z$ plane is thus

$$
E^{(m \omega)}(x, y, z)=\int_{\text {crystal }} E^{(m \omega)}\left(x, y, z, z^{\prime}\right) \mathrm{d} z^{\prime},
$$

where the limits of the integral in Eq. (10) depend on the crystal configuration. Substituting from Eqs. (3), (6a), and (6b) into Eqs. (9) and (10), and assuming $|\Delta k| \ll m k$, where $\Delta k=m k-k_{m}=m n(\omega) k_{0}-n(m \omega) m k_{0}$ is the wave-vector mismatch, and $n(\omega)$ is the refractive index at frequency $\omega$, we obtain the following results for the second and thirdharmonic fields in the two configurations presented in Fig. 1.

\section{A. Second-Harmonic Beam}

The transverse components of the second-harmonic radiated field generated by the transverse components of the fundamental vector field are Gaussian beams:

$$
\begin{gathered}
E_{x x}^{2 \omega} \propto \alpha^{2} \eta_{1}^{(2)} G\left(\rho, z, k_{2}\right), \\
E_{y y}^{2 \omega} \propto \beta^{2} \eta_{1}^{(2)} G\left(\rho, z, k_{2}\right), \\
E_{x y}^{2 \omega} \propto 2 \alpha \beta \eta_{1}^{(2)} G\left(\rho, z, k_{2}\right) .
\end{gathered}
$$

The transverse components of the second-harmonic field generated by the axial component of the fundamental vector field are Hermite-Gaussian beams of orders $(1,0)$ and $(0,1)$ :

$$
\begin{aligned}
& E_{x z}^{2 \omega} \propto-2 \alpha \eta_{1}^{(2)} G\left(\rho, z, k_{2}\right) \frac{\alpha x+\beta y}{z+j z_{0}}, \\
& E_{y z}^{2 \omega} \propto-2 \beta \eta_{1}^{(2)} G\left(\rho, z, k_{2}\right) \frac{\alpha x+\beta y}{z+j z_{0}} .
\end{aligned}
$$


The axial component of the second-harmonic field generated by the axial component of the fundamental vector field is

$$
\begin{aligned}
E_{z z}^{2 \omega} \propto & G\left(\rho, z, k_{2}\right)\left[\eta_{1}^{(2)}\left(\frac{\alpha x+\beta y}{z+j z_{0}}\right)^{2}-\left(\alpha^{2}+\beta^{2}\right) \frac{\theta_{0}^{2}}{4}\right. \\
& \left.\times\left(\eta_{2}^{(2)}-\eta_{1}^{(2)} \frac{j z_{0}}{z+j z_{0}}\right)\right],
\end{aligned}
$$

where $\theta_{0}=W_{0} / z_{0}$ is the divergence angle of the fundamental Gaussian beam. For arbitrary values of $\alpha$ and $\beta$, the first term of Eq. (12c) may be expressed as a superposition of Hermite-Gaussian beams of orders $(2,0),(0,2),(1,1)$, and $(0,0)$. For a linearly polarized fundamental wave, in a rotated coordinate system with one axis parallel to the direction of polarization, this term is a superposition of Hermite-Gaussian beams of orders $(2,0)$ and $(0,0)$. The second term in Eq. (12c) is a Gaussian beam modulated by a $z$-dependent factor, which includes a term with excess Gouy phase. This term vanishes when the fundamental wave is circularly polarized (i.e., $\beta= \pm j \alpha$, so that $\alpha^{2}$ $\left.+\beta^{2}=0\right)$.

In the above expressions, the coefficients

$$
\eta_{1}^{(2)}=j \int_{z_{1}}^{z_{2}} \mathrm{~d} z^{\prime} \frac{e^{-j \Delta k z^{\prime}}}{z^{\prime}+j z_{0}}, \quad \eta_{2}^{(2)}=-z_{0} \int_{z_{1}}^{z_{2}} \mathrm{~d} z^{\prime} \frac{e^{-j \Delta k z^{\prime}}}{\left(z^{\prime}+j z_{0}\right)^{2}}
$$

are geometrical factors that determine the magnitudes of the various SHG components, as will be discussed in Sec- tion 4. Here, the limits of the integral in Eqs. (13) are $\left(z_{1}=-L / 2, z_{2}=L / 2\right)$ in the centered configuration and $\left(z_{1}\right.$ $\left.=0, z_{2}=L\right)$ in the offset configuration. Note that in the far field, i.e., $z \gg z_{0}$, the term proportional to $\eta_{2}^{(2)}$ in relation (12c) dominates.

The spatial distributions of the optical intensity $I^{2 \omega}$ $=\left|E^{2 \omega}(\rho, z)\right|^{2}$ for each of the components of the SHG field are illustrated in Fig. 2(a) for a beam linearly polarized in the $x$ direction, i.e., $\alpha=1$ and $\beta=0$. In this case, the peak magnitudes of the various components, all occurring at $y=0$, are as follows:

(i) The peak magnitude of $E_{x x}^{2 \omega}$ occurs at $x=0$ and equals $\eta_{1}^{(2)} G_{0}$.

(ii) The peak magnitude of $E_{x z}^{2 \omega}$ occurs at $x=W(z) / \sqrt{2}$ and equals $0.86 \theta_{0} \eta_{1}^{(2)} G_{0}$.

(iii) The peak magnitude of $E_{z z}^{2 \omega}$ occurs at $x=0$ and equals $0.25 \theta_{0}^{2} \eta_{2}^{(2)} G_{0}$ in the far field $\left(z \gg z_{0}\right)$.

Since $\theta_{0} \ll 1$ for a paraxial beam, and since the factors $\eta_{1}^{(2)}$ and $\eta_{2}^{(2)}$ are of the same order of magnitude, as is shown in Section 4 , the peak magnitudes of $E_{x x}^{2 \omega}, E_{x z}^{2 \omega}$, and $E_{z z}^{2 \omega}$ are of the relative orders of $1, \theta_{0}$, and $\theta_{0}^{2}$, respectively.

\section{B. Third-Harmonic Beam}

There are ten components of the third-harmonic field. The transverse third-harmonic components generated by the transverse components of the fundamental vector field are Gaussian beams:

$$
E_{x x x}^{3 \omega} \propto-\alpha^{3} \eta_{1}^{(3)} G\left(\rho, z, k_{3}\right),
$$

$(a)$
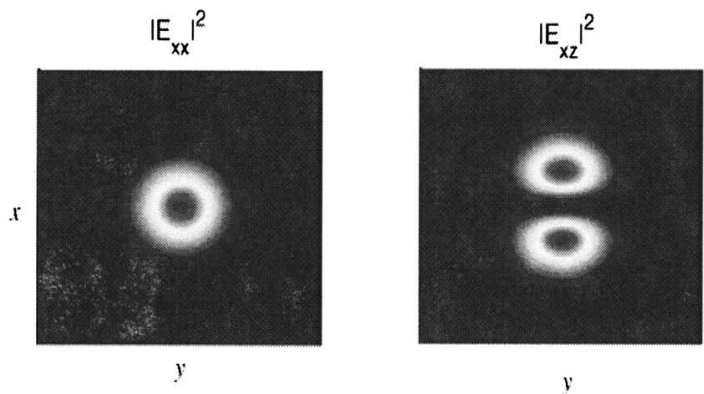

$\left|E_{x x x}\right|^{2}$

(b)

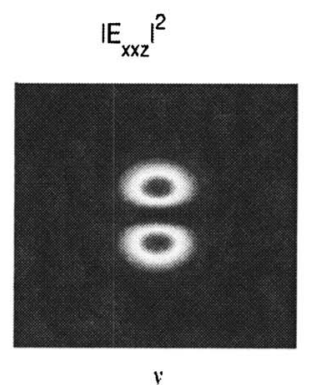

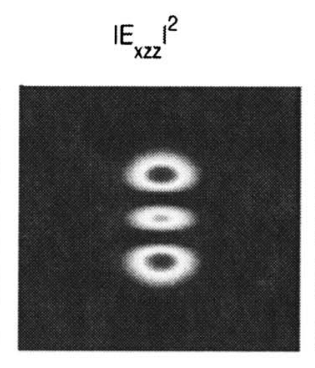

$y$

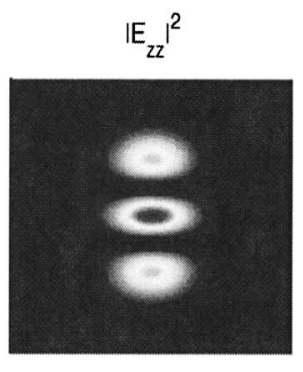

$y$

Fig. 2. (a) Optical intensities of second-harmonic components at $z=10 z_{0}$ for an offset crystal with $L=2 z_{0}$, i.e., $\xi=1$. The fundamental wave is assumed to be linearly polarized in the $x$ direction, so that $\alpha=1$ and $\beta=0$. The angle of divergence of the fundamental beam is $\theta_{0}=67 \mathrm{mrad}=3.8^{\circ}$. The $x x$ component is Gaussian, the $x z$ component is Hermite-Gaussian of order $(1,0)$, and the $z z$ component is a superposition of Hermite-Gaussian functions of orders $(0,0)$ and $(2,0)$. (b) Optical intensities of third-harmonic components at $z=10 z_{0}$ for an offset crystal with $L=2 z_{0}$, i.e., $\xi=1$. The fundamental wave is assumed to be linearly polarized in the $x$ direction, so that $\alpha=1$ and $\beta$ $=0$. The angle of divergence of the fundamental beam is $\theta_{0}=67 \mathrm{mrad}=3.8^{\circ}$. The $x x x$ component is Gaussian, the $x x z$ component is Hermite-Gaussian of order $(1,0)$, and the $x z z$ component is a superposition of Hermite-Gaussian functions of order $(0,0)$ and $(2,0)$. The $z z z$ component is a sum of Hermite-Gaussian functions of order $(1,0)$ and $(3,0)$. 


$$
\begin{gathered}
E_{y y y}^{3 \omega} \propto-\beta^{3} \eta_{1}^{(3)} G\left(\rho, z, k_{3}\right), \\
E_{x y y}^{3 \omega} \propto-3 \alpha \beta^{2} \eta_{1}^{(3)} G\left(\rho, z, k_{3}\right), \\
E_{x x y}^{3 \omega} \propto-3 \alpha^{2} \beta \eta_{1}^{(3)} G\left(\rho, z, k_{3}\right) .
\end{gathered}
$$

The transverse third-harmonic components generated by combinations of transverse and axial components of the fundamental vector field are Hermite-Gaussian beams of order $(1,0)$ and $(0,1)$ :

$$
\begin{aligned}
& E_{x x z}^{3 \omega} \propto 3 \alpha^{2} \eta_{1}^{(3)} G\left(\rho, z, k_{3}\right) \frac{\alpha x+\beta y}{z+j z_{0}}, \\
& E_{y y z}^{3 \omega} \propto 3 \beta^{2} \eta_{1}^{(3)} G\left(\rho, z, k_{3}\right) \frac{\alpha x+\beta y}{z+j z_{0}}, \\
& E_{x y z}^{3 \omega} \propto 3 \alpha \beta \eta_{1}^{(3)} G\left(\rho, z, k_{3}\right) \frac{\alpha x+\beta y}{z+j z_{0}} .
\end{aligned}
$$

The transverse third-harmonic components generated by the axial component of the fundamental vector field are

$$
\begin{aligned}
E_{x z z}^{3 \omega} \propto & -3 \alpha G\left(\rho, z, k_{3}\right)\left[\eta_{1}^{(3)}\left(\frac{\alpha x+\beta y}{z+j z_{0}}\right)^{2}-\left(\alpha^{2}+\beta^{2}\right) \frac{\theta_{0}^{2}}{6}\right. \\
& \left.\times\left(\eta_{2}^{(3)}-\eta_{1}^{(3)} \frac{j z_{0}}{z+j z_{0}}\right)\right], \\
E_{y z z}^{3 \omega} \propto & -3 \beta G\left(\rho, z, k_{3}\right)\left[\eta_{1}^{(3)}\left(\frac{\alpha x+\beta y}{z+j z_{0}}\right)^{2}-\left(\alpha^{2}+\beta^{2}\right) \frac{\theta_{0}^{2}}{6}\right. \\
& \left.\times\left(\eta_{2}^{(3)}-\eta_{1}^{(3)} \frac{j z_{0}}{z+j z_{0}}\right)\right] .
\end{aligned}
$$

The first terms of Eqs. (14h) and (14i) are superpositions of Hermite-Gaussian beams of orders $(2,0),(0,2),(1,1)$, and $(0,0)$. The second terms are Gaussian beams modulated by a $z$-dependent factor.

Finally, the axial third-harmonic component generated by the axial component of the fundamental vector field is

$$
\begin{aligned}
E_{z z z}^{3 \omega} \propto & G\left(\rho, z, k_{3}\right)\left\{\eta_{1}^{(3)}\left(\frac{\alpha x+\beta y}{z+j z_{0}}\right)^{3}-\left(\alpha^{2}+\beta^{2}\right) \frac{\theta_{0}^{2}}{2}\right. \\
& \left.\times\left(\eta_{2}^{(3)}-\eta_{1}^{(3)} \frac{j z_{0}}{z+j z_{0}}\right)\left(\frac{\alpha x+\beta y}{z+j z_{0}}\right)\right\} .
\end{aligned}
$$

The first term of Eq. (14j) is a superposition of HermiteGaussian beams of orders $(3,0),(0,3),(2,1),(1,2),(1,0)$, and $(0,1)$. The second term is a superposition of HermiteGaussian beams of orders $(1,0)$ and $(0,1)$, modulated by a $z$-dependent factor.

The magnitudes of these components are determined by the geometrical factors

$$
\eta_{1}^{(3)}=-z_{0} \int_{z_{1}}^{z_{2}} \mathrm{~d} z^{\prime} \frac{e^{-j \Delta k z^{\prime}}}{\left(z^{\prime}+j z_{0}\right)^{2}}, \quad \eta_{2}^{(3)}=-j z_{0}^{2} \int_{z_{1}}^{z_{2}} \mathrm{~d} z^{\prime} \frac{e^{-j \Delta k z^{\prime}}}{\left(z^{\prime}+j z_{0}\right)^{3}}
$$

Note that in the far field, i.e., $z \gg z_{0}$, the terms proportional to $\eta_{2}^{(3)}$ in Eqs. (14h)-(14j) dominate. It is also interesting to note that the THG coefficient $\eta_{1}^{(3)}$ is equal to the SHG coefficient $\eta_{2}^{(2)}$ so that the axial component of SHG is governed by the same geometrical factor as that of the transverse component of THG.

The spatial distributions of the optical intensity $I^{3 \omega}$ $=\left|E^{3 \omega}(\rho, z)\right|^{2}$ for some of the components of the THG field are illustrated in Fig. 2(b) for a beam linearly polarized in the $x$ direction, i.e., $\alpha=1$ and $\beta=0$. In this case, the peak magnitudes of the various components, all occurring at $y=0$, are as follows:

(i) The peak magnitude of $E_{x x x}^{3 \omega}$ occurs at $x=0$ and equals $\eta_{1}^{(3)} G_{0}$.

(ii) The peak magnitude of $E_{x x z}^{3 \omega}$ occurs at $x=W(z) / \sqrt{2}$, $y=0$, and equals $1.29 \theta_{0} \eta_{1}^{(3)} G_{0}$.

(iii) The peak magnitude of $E_{x z z}^{3 \omega}$ occurs at $x=0$ and equals $0.5 \theta_{0}^{2} \eta_{2}^{(3)} G_{0}$ in the far field $\left(z \gg z_{0}\right)$.

(iv) The component $E_{z z z}^{3 \omega}$ has its peak at $x=W(z) / \sqrt{2}$ and equals $0.23 \theta_{0}^{3} \eta_{2}^{(3)} G_{0}$ in the far field $\left(z \gg z_{0}\right)$.

The peak magnitudes of $E_{x x x}^{3 \omega}, E_{x x z}^{3 \omega}, E_{x z z}^{3 \omega}$, and $E_{z z z}^{3 \omega}$ are therefore of the relative orders of $1, \theta_{0}, \theta_{0}^{2}$, and $\theta_{0}^{3}$, respectively.

The preceding result may be generalized to the $m$ th harmonic process. An $m$ th harmonic generation process involving $\ell$ axial components and $m$ - $\ell$ transverse components of the fundamental field generates a field with a magnitude proportional to $\theta_{0}^{1}$, so that the effect is smaller, the greater the number $\ell$. If $\theta_{0}=0.1$, for example, the effect is reduced by an order of magnitude as $\ell$ increases by one. Note, however, that the contributions of each of these nonlinear processes is also dependent on the nonlinear optical coefficients associated with the radiation process, as described by Eqs. (6a) and (6b) for the second- and third-harmonic generation processes, respectively. Since the magnitudes of nonlinear coefficients of an anisotropic crystal may differ by many orders of magnitude, contributions from terms of greater $\ell$ may end up having greater contributions to the overall process.

\section{EFFICIENCY OF HARMONIC GENERATION}

In general, the geometrical factors $\eta_{1}^{(m)}$ and $\eta_{2}^{(m)}$ in Eqs. (13) and (15) are

$$
\begin{gathered}
\eta_{1}^{(m)}=\left(j z_{0}\right)^{m-2} \int_{z_{1}}^{z_{2}} \mathrm{~d} z^{\prime} \frac{j e^{-j \Delta k z^{\prime}}}{\left(z^{\prime}+j z_{0}\right)^{m-1}}, \\
\eta_{2}^{(m)}=\left(j z_{0}\right)^{m-1} \int_{z_{1}}^{z_{2}} \mathrm{~d} z^{\prime} \frac{j e^{-j \Delta k z^{\prime}}}{\left(z^{\prime}+j z_{0}\right)^{m}} .
\end{gathered}
$$

We now evaluate the dependence of these geometrical factors $\eta_{1}^{(m)}$ and $\eta_{2}^{(m)}$ for SHG $(m=2)$ and THG $(m=3)$ on the 
ratio $\xi=L / 2 z_{0}$. These factors govern the magnitudes of the various components of SHG and THG. We consider two special cases: (1) perfect phase matching (i.e., $\Delta k=0$ ) and (2) a crystal of infinite length.

\section{A. Second-Harmonic Generation}

\section{Phase-Matched Case}

When $\Delta k=0$, the integrals in Eqs. (13) may be readily evaluated. For the centered crystal,

$$
\eta_{1}^{(2)}=2 \tan ^{-1} \xi, \quad \eta_{2}^{(2)}=\frac{2 \xi}{1+\xi^{2}}
$$

where $\xi=L / 2 z_{0}$. Asymptotic values of these factors in the limit $\xi \gg 1$ are $\eta_{1}^{(2)}=\pi$ and $\eta_{2}^{(2)}=2 / \xi$, respectively.

For the offset crystal $\left(z_{1}=0, z_{2}=L\right)$,

$$
\eta_{1}^{(2)}=j \ln (1-j 2 \xi), \quad \eta_{2}^{(2)}=\frac{2 \xi}{1-j 2 \xi}
$$

and the asymptotic values of these factors in the limit $\xi$ $\gg 1$ are $\eta_{1}^{(2)}=j \ln (1-j 2 \xi)$ and $\eta_{2}^{(2)}=j$. The dependence of these efficiencies on $\xi$ is shown in Fig. 3 for centered and offset crystals.

\section{Effect of Phase Mismatch}

The effect of phase mismatch on the different SHG efficiencies may be determined by numerical evaluation of Eqs. (13). Figure 4 illustrates the dependence of these efficiencies on the parameter $\chi=\Delta k z_{0}$ in the limit of an infinite crystal. The following analytical expressions are obtained in this limit in the centered configuration:

$$
\eta_{1}^{(2)}=2 \pi e^{-\chi} V(\chi), \quad \eta_{2}^{(2)}=2 \pi \chi e^{-\chi} U(\chi),
$$

where
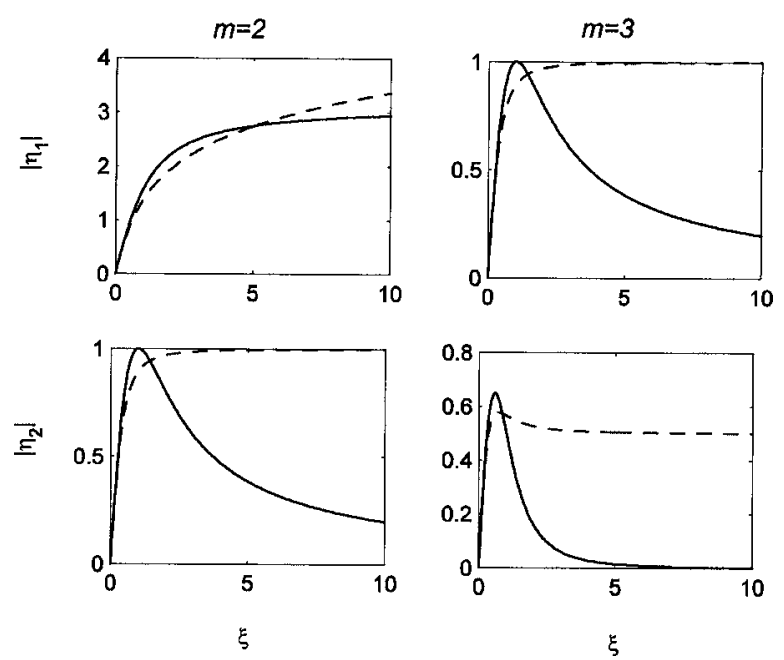

Fig. 3. Dependence of the magnitudes of the efficiency factors $\eta_{1}^{(m)}$ and $\eta_{2}^{(m)}$ on the geometrical factor $\xi=L / 2 z_{0}$ for SHG $(m=2)$ and THG $(m=3)$ in the phase-matched case with a centered crystal (solid curves) and with an offset crystal (dashed curves).
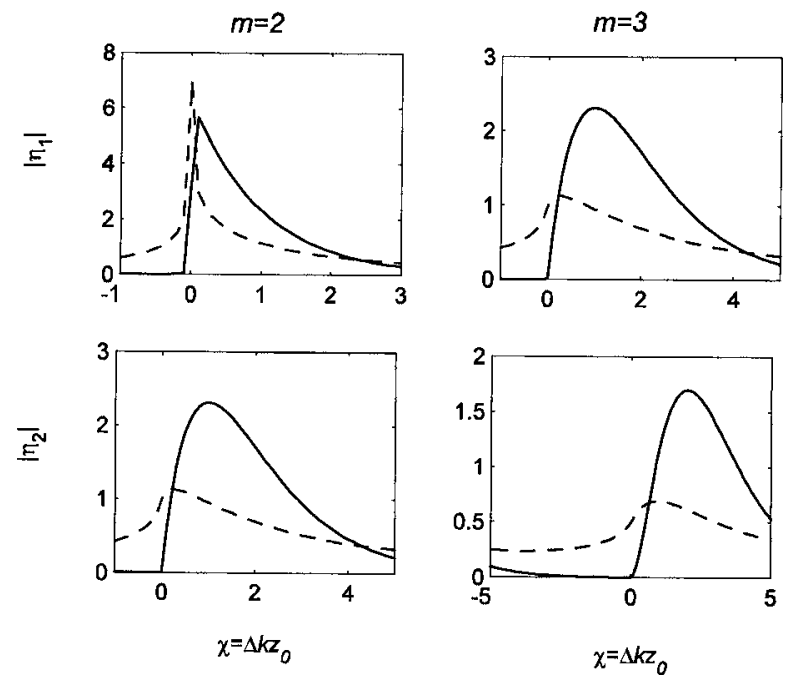

Fig. 4. Dependence of the magnitudes of the efficiency factors $\eta_{1}^{(m)}$ and $\eta_{2}^{(m)}$ on the phase mismatch factor $\chi=\Delta k z_{0}$ for SHG $(m$ $=2)$ and THG $(m=3)$ assuming a long crystal $\left(\xi=L / 2 z_{0}=10^{3}\right)$ that is centered (solid curves) or offset (dashed curves).

$$
U(\chi)=\left\{\begin{array}{ll}
0, & \chi \leqslant 0 \\
1, & \chi>0
\end{array}, \quad V(\chi)= \begin{cases}0, & \chi<0 \\
\frac{1}{2}, & \chi=0 \\
1, & \chi>0\end{cases}\right.
$$

These expressions are consistent with Eqs. (17) in the case of $\chi=0$ in the limiting case $\xi \rightarrow \infty$.

\section{B. Third-Harmonic Generation}

\section{Phase-Matched Case}

In the phase-matched case, $\Delta k=0$, and for the centered crystal,

$$
\eta_{1}^{(3)}=\frac{2 \xi}{1+\xi^{2}}, \quad \eta_{2}^{(3)}=\frac{2 \xi}{\left(1+\xi^{2}\right)^{2}}
$$

In the limit $\xi \gg 1, \eta_{1}^{(3)}=2 / \xi, \eta_{2}^{(3)}=2 / \xi^{3}$.

For the offset crystal $\left(L_{1}=0, L_{2}=L\right)$,

$$
\eta_{1}^{(3)}=\frac{2 \xi}{1-j 2 \xi}, \quad \eta_{2}^{(3)}=\frac{2 \xi(1-j \xi)}{(1-j 2 \xi)^{2}} .
$$

In the limit $\xi \gg 1, \eta_{1}^{(3)}=j, \eta_{2}^{(3)}=j / 2$.

\section{Effect of Phase Mismatch}

The effect of phase mismatch on the different THG efficiencies may be determined by numerical evaluation of Eqs. (15). Figure 4 illustrates the dependence of these efficiencies on the parameter $\chi=\Delta k z_{0}$ in the limit of an infinite crystal. The following analytical expressions are obtained in this limit in the centered configuration:

$$
\eta_{1}^{(3)}=2 \pi \chi e^{-\chi} U(\chi), \quad \eta_{2}^{(3)}=\pi \chi^{2} e^{-\chi} U(\chi)
$$

These expressions are consistent with Eqs. (20) in the case $\chi=0$ in the limiting case $\xi \rightarrow \infty$. 


\section{Dependence of Efficiency Factors on Geometry and Phase Mismatch}

Figure 3 illustrates the dependence of $\eta_{1}^{(m)}$ and $\eta_{2}^{(m)}$ on the ratio $\xi=L / 2 z_{0}$ in the centered and offset configurations shown in Fig. 1 for SHG $(m=2)$ and THG $(m=3)$. Expressions for the factors $\eta_{1}^{(m)}$, which govern the transverse components, have been previously established and their dependences on the ratio $\xi$ have been set forth in the literature (see, for example, Ref. 6). The factor $\eta_{1}^{(2)}$ increases steadily with increasing $\xi$ in SHG for both configurations. The decrease of $\eta_{1}^{(3)}$ with increasing $\xi$ in the THG centered configuration is a result of the phase mismatch induced by the Gouy phase shift that causes destructive interference of the THG radiation from the various parts of the sample.

The factors $\eta_{2}^{(m)}$, which govern the axial components, particularly in the far field, behave differently. In SHG, $\eta_{2}^{(2)}$ (which equals $\eta_{1}^{(3)}$ ) declines with increasing $\xi$ in the centered case. This is a result of the doubled Gouy phase exhibited in the axially polarized components. An even sharper decline is exhibited in the THG factor $\eta_{2}^{(3)}$. In all cases, the use of an offset crystal alleviates such a decline, in which case the efficiencies of all harmonic components increase with increasing $\xi$ before eventually saturating.

The different efficiencies of SHG and THG are plotted in Fig. 4 as functions of the phase mismatch factor $\chi$ $=\Delta k z_{0}$ for a long crystal $\left(\xi=L / 2 z_{0} \gg 1\right)$ in the centered and offset configurations. These efficiencies are not symmetric functions of $\Delta k$ and their peak values do not necessarily occur at $\Delta k=0$, as is the case when the fundamental wave is a plane wave. SHG and THG diminish sharply for negative values of $\Delta k$, but are more tolerant to positive $\Delta k$.

\section{CONCLUSION}

The Gouy phase associated with the wavefront curvature of a focused optical beam plays an important role in harmonic generation; indeed, it is well known that it is responsible for the diminution of THG for a centered crystal. However, wavefront curvature is also accompanied by an axially polarized component in a focused beam. For a Gaussian beam, this component takes the form of Hermite-Gaussian (1,0) and $(0,1)$ modes, which have twice the Gouy phase of the transversely polarized components. Harmonic generation contributed by the axially polarized component creates SHG and THG beams that are superpositions of low-order Hermite-Gaussian modes. The efficiency of an $m$ th harmonic generation process involving $\ell$ axial and $m-\ell$ transverse components is proportional to the beam divergence angle raised to the $\ell$ th power, and is therefore smaller when a large number of axial components are involved. The harmonic generation efficiency is also proportional to geometrical factors that diminish with crystal size in the centered case, for both SHG and THG; the effect is more pronounced for THG than for SHG. On the other hand, the behavior of these geometrical factors with phase mismatch for the axially polarized component is not unlike that for the transversely polarized components.
Although our analytical model was based on a vector Gaussian beam excitation, conclusions regarding the spatial distributions and the geometrical factors associated with the polarization components of the harmonic beams are expected to apply to a more tightly focused excitation beam, since the Gaussian model captures the basic geometry of a focused vector beam. However, the results are expected to be altered for apertured excitation beams with significantly different non-Gaussian distributions since the aperture transverse distribution controls the polarization state in the focal region. ${ }^{23}$

\section{ACKNOWLEDGMENTS}

This work was supported by the Fulbright Program and the Spanish Ministry of Education and Science; the National Science Foundation (NSF) through the Center for Subsurface Imaging Systems (CenSSIS), an NSF Engineering Research Center; a U.S. Army Research Office (ARO) Multidisciplinary University Research Initiative (MURI) grant; and the David \& Lucile Packard Foundation. We are grateful to A. Abouraddy for valuable discussions.

S. Carrasco's e-mail address is silvia.carrasco@icfo.es.

\section{REFERENCES}

1. D. A. Kleinman, A. Ashkin, and G. D. Boyd, "Second harmonic generation of light by focused laser beams," Phys. Rev. 145, 338-379 (1966).

2. G. D. Boyd and D. A. Kleinman, "Parametric interaction of focused Gaussian light beams," J. Appl. Phys. 39, 3597-3639 (1968).

3. J. F. Ward and G. H. C. New, "Optical third harmonic generation in gases by a focused laser beam," Phys. Rev. 185, 57-72 (1969).

4. G. C. Bjorklund, "Effects of focusing on third-order nonlinear processes in isotropic media," IEEE J. Quantum Electron. QE-11, 287-296 (1975).

5. R. B. Miles and S. E. Harris, "Optical third-harmonic generation in alkali metal vapors," IEEE J. Quantum Electron. QE-9, 470-484 (1973).

6. R. W. Boyd, Nonlinear Optics, 2nd ed. (Academic, 2003), Chap. 2.

7. M. Gouy, "Sur la propagation anomale des ondes," C. R. Hebd. Seances Acad. Sci. 111, 33-40 (1890).

8. A. E. Siegman, Lasers (University Science, 1986).

9. R. Gauderon, P. B. Lukins, and C. J. R. Sheppard, "Threedimensional second-harmonic generation imaging with femtosecond laser pulses," Opt. Lett. 23, 1209-1211 (1998).

10. P. J. Campagnola, M.-de. Wei, A. Lewis, and L. M. Loew, "High-resolution nonlinear optical imaging of live cells by second harmonic generation," Biophys. J. 77, 3341-3349 (1999).

11. L. Moreaux, O. Sandre, and J. Mertz, "Membrane imaging by second-harmonic generation microscopy," J. Opt. Soc. Am. B 17, 1685-1694 (2000).

12. Y. Barad, H. Eisenberg, M. Horowitz, and Y. Silberberg, "Nonlinear scanning laser microscopy by third harmonic generation," Appl. Phys. Lett. 70, 922-924 (1997).

13. J. A. Squier, M. Muller, G. J. Brakenhoff, and K. R. Wilson, "Third harmonic generation microscopy," Opt. Express 3, 315-324 (1998).

14. M. Muller, J. A. Squier, K. R. Wilson, and G. J. Brakenhoff, "3D microscopy of transparent object using third harmonic generation," J. Microsc. 191, 266-274 (1998).

15. D. Yelin and Y. Silberberg, "Laser scanning third harmonic generation in biology," Opt. Express 5, 169-175 (1999).

16. D. Oron and Y. Silberberg, "Third-harmonic generation 
with cylindrical Gaussian beams," J. Opt. Soc. Am. B 21, 1964-1968 (2004).

17. D. Debarre, W. Supatto, and E. Beaurepatre, "Structure sensitivity in third-harmonic generation microscopy," Opt. Lett. 30, 2134-2136 (2005).

18. J.-X. Cheng and X. S. Xie, "Green's function formulation for third-harmonic generation microscopy," J. Opt. Soc. Am. B 19, 1604-1610 (2002).

19. E. Yew and C. Sheppard, "Effects of axial field components on second harmonic generation microscopy," Opt. Express 14, 1167-1174 (2006).
20. H. A. Haus, Waves and Fields in Optoelectronics (PrenticeHall, 1984).

21. R. W. Hellwarth, "Third-order optical susceptibilities of liquids and solids," Prog. Quantum Electron. 5, 1-68 (1977).

22. B. E. A. Saleh and M. C. Teich, Fundamentals of Photonics (Wiley, 1991), Chaps. 3 and 19.

23. A. F. Abouraddy and K. C. Toussaint, Jr., "Threedimensional polarization control in microscopy," Phys. Rev. Lett. 96, 153901 (2006). 